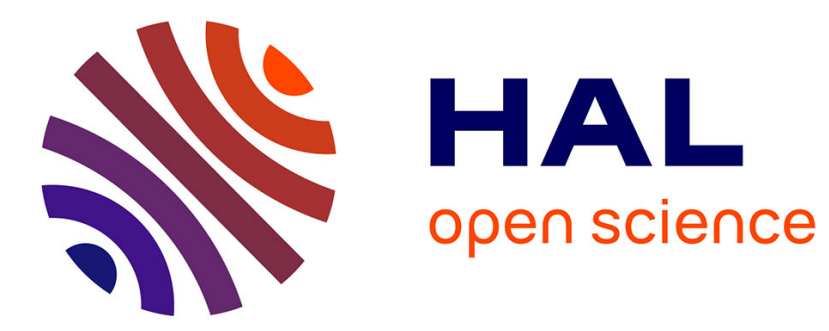

\title{
L'hydraulique pastorale, un bienfait pour les éleveurs du Sahel?
}

Catherine Baroin

\section{To cite this version:}

Catherine Baroin. L'hydraulique pastorale, un bienfait pour les éleveurs du Sahel?. Afrique Contemporaine, 2003, 205, pp.205-224. hal-00749721

\section{HAL Id: hal-00749721 \\ https://hal.science/hal-00749721}

Submitted on 8 Nov 2012

HAL is a multi-disciplinary open access archive for the deposit and dissemination of scientific research documents, whether they are published or not. The documents may come from teaching and research institutions in France or abroad, or from public or private research centers.
L'archive ouverte pluridisciplinaire HAL, est destinée au dépôt et à la diffusion de documents scientifiques de niveau recherche, publiés ou non, émanant des établissements d'enseignement et de recherche français ou étrangers, des laboratoires publics ou privés. 


\section{L'hydraulique pastorale, un bienfait pour les éleveurs du Sahel ?}

Catherine Baroin *

On désigne par "hydraulique pastorale" la politique de multiplication des points d'eau et de modernisation de l'abreuvage, qui fut mise en ouvre au Sahel à partir des années 1950. Son objectif était de développer et d'intensifier l'élevage extensif en jouant sur un facteur essentiel, l'accès à l'eau. Du Sénégal au Soudan, les populations pastorales concernées étaient diverses : Maures, Peuls, Touaregs, Toubou, ainsi que de nombreuses tribus arabes plus ou moins métissées, nomadisant surtout dans l'est du Tchad et au Soudan.

\section{Un projet et ses origines}

Techniquement, la création de stations de pompage mécaniques ou de forages artésiens ne posait pas de problèmes, et cette politique fut appliquée à vaste échelle. Elle eut des effets immédiats et contribua sans conteste au développement de l'élevage sahélien. Mais elle provoqua en même temps de nombreuses difficultés dont nous retracerons ici la logique. Les dysfonctionnements entraînés par cette politique trop exclusivement fondée sur des considérations techniques ont mis en lumière, en les bousculant, des aspects essentiels de l'organisation des sociétés africaines concernées.

* Anthropologue, Centre national de la recherche scientifique (CNRS), UMR 7041, Nanterre. 


\section{Le milieu sahélien précolonial, zone d'élevage extensif}

Pour retracer l'impact de l'hydraulique pastorale, il est nécessaire de rappeler d'abord le contexte dans lequel s'inscrivait cette ambition, c'est-à-dire les contraintes du milieu naturel sahélien et de son mode d'exploitation.

Comme on le sait, le Sahel longe en un large bandeau le sud du Sahara, sur environ $4000 \mathrm{~km}$ d'ouest en est, de Dakar à la frontière soudanienne. Dans sa partie nord, les pluies (entre 150 et $400 \mathrm{~mm}$ par an) sont insuffisantes et trop irrégulières pour cultiver. Elles sont plus abondantes à mesure que l'on descend vers le sud, où le couvert végétal devient plus dense : il se compose de graminées et d'arbres qui font du Sahel une zone d'élevage. Le pâturage herbeux et arboré nourrit les dromadaires (abusivement appelés "chameaux") au nord, et le petit bétail et les bovins qui s'y ajoutent au sud.

Les pluies se concentrent sur une courte saison, de juillet à septembre. Irrégulières dans le temps (avec de fortes variations annuelles) comme dans l'espace, elles sont si localisées que deux lieux voisins peuvent en recevoir, en une saison, des quantités très différentes. La présence de pâturage étant tributaire de ces pluies, le cheptel doit se déplacer pour profiter des meilleurs emplacements. La mobilité du bétail et des hommes est indispensable et l'élevage ne peut être qu'extensif.

La saison des pluies renouvelle la flore chaque année. Les graminées reverdissent et, dans les zones méridionales, des mares temporaires remplissent le fond des cuvettes argileuses. Puis l'herbe prend rapidement une teinte blond pâle et les mares s'assèchent jusqu'à l'année suivante. L'herbe sèche que broutent les animaux se raréfie peu à peu, et les branches et les fruits des arbres fournissent un complément alimentaire indispensable au bétail, surtout lors des chaleurs intenses d'avril et mai. C'est l'éprouvante période de soudure et tous attendent comme une bénédiction la tombée des premières pluies. 
La saison des pluies est la saison faste. Les bêtes reprennent des forces en pâturant l'herbe verte, le lait est plus abondant. L'abreuvage est moins astreignant et devient même inutile quand les animaux boivent d'eux-mêmes aux mares temporaires du voisinage, auprès desquelles se regroupent de nombreux troupeaux. Pour un temps, les éleveurs sont alors dispensés de cette pénible corvée. Mais, dès que les mares s'assèchent, l'abreuvage régulier des bovins redevient indispensable jusqu'aux prochaines pluies. Les chamelles peuvent s'éloigner des points d'eau plus que les vaches ${ }^{1}$, car elles nécessitent un abreuvage moins fréquent et peuvent s'en dispenser totalement à la saison froide où les bergers s'éloignent des campements pour les conduire sur les pâturages du désert. Mais, en dehors de ces périodes, l'élevage est tributaire des puits.

Ceux-ci, dans le système traditionnel, sont creusés par des spécialistes. Si le sol est dur, ils peuvent être très profonds². Mais, s'il est sableux, l'effondrement menace et les puits sont consolidés par un coffrage de bois sur toute leur hauteur. Comme ils sont fragiles, ils ne sont creusés que si la nappe phréatique n'est pas trop profonde. Le coffrage est construit par le puisatier du haut vers le bas, à mesure qu'il creuse. Il utilise des rondins prélevés sur les arbres des environs, et bourre les interstices avec une herbe imputrescible qui retient le sable et retarde l'ensablement du puits. A l'orifice sont ensuite plantées une à trois fourches de bois, selon l'espace disponible.

L'éleveur qui vient abreuver son bétail dispose sur l'une des fourches un axe horizontal muni d'une poulie, sur laquelle tourne la corde de la puisette. Cette dernière, d'abord jetée au fond du puits, est ensuite remontée à la main, si le puits n'est pas trop profond, ou par traction animale. Dans ce cas, l'autre extrémité de la corde est fixée au poitrail d'un âne, d'un bœuf ou d'un dromadaire qui s'éloigne du puits en tirant la corde ; celle-ci tourne alors sur la poulie supportée par la fourche. La puisette est dégagée de la fourche à la main puis transvasée dans un abreuvoir proche, où les bêtes boivent, tandis que l'on relance à nouveau la puisette dans le puits.

1) Les troupeaux se composent surtout de femelles, élevées pour le lait et pour le croît.

2) Jusqu'à plus de $100 \mathrm{~m}$ au nord de Tombouctou (Mali), de 50 à $90 \mathrm{~m}$ au sud d'In Gall (Niger) (Bernus, 1989, p. 95). 
Ce travail éprouvant revient fréquemment aux adolescents, garçons ou filles, quand les familles sont dépourvues d'esclave ou de berger rémunéré. L'animal qui remonte la corde est guidé par un jeune enfant, tandis qu'un autre surveille les tours d'abreuvage. L'opération se poursuit, puisette après puisette, pendant des heures, dans la poussière et sous le soleil, jusqu'à ce que toutes les bêtes du troupeau familial soient abreuvées.

Cette technique d'abreuvage n'est pas seulement harassante, elle est peu efficace. Le débit souvent faible de ces puits, l'attente nécessaire pour que la puisette se remplisse, le temps pris par sa remontée limitent le nombre d'animaux qu'un berger peut abreuver, même lorsqu'il prolonge son travail tard dans la nuit. De plus, on ne peut abreuver que deux ou trois troupeaux à la fois : l'étroitesse du puits et le petit nombre des fourches limitent celui des puisettes utilisables simultanément. Autre défaut, ces puits traditionnels durent peu. Le va-et-vient des puisettes, auquel s'ajoute le ruissellement d'eaux fangeuses à la saison des pluies, provoquent leur effondrement après deux ou trois ans. Les troupeaux sont alors conduits ailleurs, en attendant qu'un nouveau puits soit creusé, le plus souvent à côté du précédent. Mais, quand le sol est trop dur ou la nappe phréatique trop profonde, les techniques traditionnelles sont impuissantes, ce qui laisse de vastes espaces inexploités.

\section{Lélevage dans le contexte colonial}

De prime abord, la conquête coloniale ne changea guère la vie quotidienne des éleveurs du Sahel. Leurs troupeaux paissaient loin des centres administratifs et leurs techniques pastorales n'étaient pas modifiées. Cependant, cette situation politique nouvelle introduisit deux facteurs de changement, qui vinrent peser sur le mode de vie pastoral et amenèrent l'administration française à souhaiter développer l'élevage sahélien.

Le premier de ces facteurs fut la pacification qui, certes, demeura lente et progressive, mais devint une source radicale de changement. Auparavant, l'insécurité était la règle. Les razzias, les vols incessants de bétail, accompagnés ou non de capture de femmes et d'enfants, entraînaient dans leur sillage vendettas, règlements de compte, 
représailles diverses. Il fallait mobiliser de grandes énergies contre ces risques. Avec la pacification, les populations sahéliennes purent mieux se consacrer à la pratique de l'élevage, posséder de grands troupeaux qui nécessitaient moins de surveillance, la répression des vols de bétail étant désormais prise en charge par le colonisateur. Cette situation fut donc propice au développement économique.

Le second facteur essentiel de changement fut l'abolition de l'esclavage, mise en œuvre au Sahel à partir de 1906, après une injonction de la conférence de Bruxelles (1889-1890) ${ }^{3}$. La traite des esclaves, qui se pratiquait du sud vers le nord du Sahara, fut alors vite enrayée et prit fin vers 1910. Des milliers d'esclaves furent libérés, mais beaucoup d'autres, nés en servitude, restèrent chez leurs anciens maîtres à tel point qu'aujourd'hui encore l'esclavage domestique n'a pas entièrement disparu du Sahel ${ }^{4}$. Mais le nombre des "captifs" a si fortement chuté que le confort des familles s'en est beaucoup ressenti. En l'absence des esclaves, la main-d'œuvre familiale était parfois insuffisante pour abreuver les troupeaux à leur place.

Par ailleurs, l'essor démographique devenait sensible, dans les villes surtout, mais aussi en brousse, où l'accroissement du cheptel fut conforté dans les années 1950 et 1960 par une pluviométrie très favorable. Or l'élevage était la grande et unique richesse du Sahel, et son développement s'imposa comme un objectif évident. Il permettrait de répondre aux besoins en viande d'une population urbaine croissante, et de développer également un marché d'exportations.

Dans les années 1950, le développement de l'élevage passait ainsi par trois grandes mesures :

- lutter contre la mortalité du bétail par des campagnes massives de vaccination $^{6}$; 
- améliorer l'efficacité de l'abreuvage grâce à des installations hydrauliques modernes (qui remédieraient en même temps à la pénurie de main-d'œuvre provoquée par le départ des esclaves);

- augmenter les surfaces pâturables en créant des points d'eau dans les zones jusqu'alors inaccessibles faute de puits.

La vaccination du bétail relevait des services vétérinaires de l'administration coloniale, tandis que l'aménagement des puits était organisé dans un cadre spécifique, celui du Service de l'hydraulique de l'Afrique occidentale française (AOF), qui fut créé en septembre 1949 sous la direction de Pierre Merlin, polytechnicien et ingénieur des Ponts et Chaussées. Ce service connut un développement rapide : en 3 ou 4 années, une centaine de Français, dont environ 15 ingénieurs, furent recrutés, et des bureaux implantés dans chaque territoire colonial. Le développement de l'hydraulique resta centralisé dans ce service jusqu'en 1958, date des autonomies internes. Puis les Etats africains, devenus pleinement indépendants deux ans plus tard, poursuivirent cette politique en créant chacun, dans son cadre national, un service ou même un ministère en charge de l'hydraulique?.

\section{Les objectifs de l'hydraulique pastorale}

L'hydraulique pastorale, ayant débuté vigoureusement dans les années 1950, s'accentua encore après les indépendances, dans les années 1960. Elle avait deux objectifs : moderniser l'abreuvage grâce à des installations modernes, et augmenter les surfaces de pâturage en creusant des puits nouveaux dans les espaces jusqu'alors inexploités. Trois types de puits furent construits, selon les caractéristiques de la nappe d'eau souterraine : des puits cimentés, des stations de pompage motorisé et des puits artésiens.

Les puits cimentés étaient les plus simples à réaliser et devinrent de ce fait les plus nombreux. Ils furent construits en remplacement parfois des puits traditionnels, lorsque l'eau était peu profonde $(35 \mathrm{~m}$ au maximum), et équipés comme ces derniers de fourches pour les poulies. La remontée de l'eau s'opérait, de la même manière, au moyen

7) Selon une information orale de Pierre Merlin, le 12 décembre 2002. 
d'une corde et d'une puisette. Les calculs de divers spécialistes de l'élevage - tels Merlin (1951) puis Receveur (1959-1960, 1965, 1975) - avaient déterminé avec une grande précision la distance optimale entre ces puits. D'après Receveur, elle devait être de $24 \mathrm{~km}^{8}$.

Quand la nappe phréatique était nettement plus profonde (de 200 à $600 \mathrm{~m}$ en général) et que le puits cimenté n'était donc pas envisageable, on installa des stations de pompage. L'eau atteinte par forage est alors remontée grâce à un moteur abrité sous un hangar. Pour la stocker, un château d'eau est construit à proximité, d'où elle est redistribuée dans des abreuvoirs cimentés. La création de telles stations coûtait beaucoup plus cher que des puits cimentés, et leur fonctionnement entraînait quelques dépenses : l'achat régulier de gazole et les frais d'entretien du moteur. En contrepartie, la capacité d'abreuvage était bien plus élevée. Conscients de la menace que la surcharge animale ferait peser sur les pâturages environnants, les responsables du développement décidèrent que ces stations seraient espacées d'environ $40 \mathrm{~km}$ les unes des autres. De nombreux forages furent ainsi réalisés, d'abord au Sénégal sur une ligne allant de Linguère à Matam, puis au Niger, entre Tahoua et Agadès?

Enfin, si la nappe d'eau souterraine est sous pression, il n'est plus besoin d'aucun moteur car le forage fait jaillir de lui-même l'eau en surface : c'est le puits artésien, qui déverse en continu une eau d'excellente qualité. Si celle-ci est abondante, elle ne sert pas seulement à abreuver le bétail, mais irrigue aussi des cultures, comme par exemple au Niger à l'ouest d'Agadès ${ }^{10}$.

\section{Les problèmes rencontrés}

Ces trois types de réalisations techniques présentaient un avantage commun : celui d'offrir une eau plus propre que celle des puits traditionnels, ce qui ne pouvait être que bénéfique pour la santé du

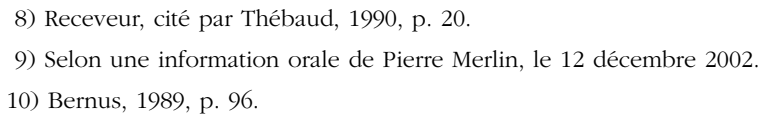


cheptel. Par ailleurs les avantages et inconvénients respectifs de ces installations étaient différents, comme on le verra à l'examen successif des points de vue technique, écologique et social.

\section{L'aspect technique}

Le suivi technique de ces points d'eau dépendait, bien sûr, de la nature de l'installation.

Les puits cimentés sont les plus semblables aux puits traditionnels, et n'entraînent aucun changement dans la façon de remonter l'eau. Cependant le diamètre du puits est un peu plus large, ce qui permet d'installer une fourche supplémentaire, et donc d'augmenter un peu le nombre d'animaux abreuvés. En outre la longévité des puits cimentés est bien supérieure à celle des puits indigènes, ils sont plus solides et ne s'effondrent pas. Par contre, ils peuvent s'ensabler et deviennent alors inutilisables. Pour les dégager, il est nécessaire de travailler en apnée au fond de l'eau, et il a fallu former des spécialistes à cette technique, comme ce fut le cas par exemple à Diffa au Niger ${ }^{11}$. Mais ce type d'intervention reste épisodique, et les puits cimentés ne nécessitent par ailleurs aucun entretien. C'est leur gros avantage, par rapport aux stations de pompage.

Ces dernières exigent une maintenance plus élaborée : l'intervention périodique d'un technicien étranger au milieu pastoral, le mécanicien, pour l'entretien du moteur et sa réparation en cas de panne. En outre, pour assurer l'approvisionnement régulier de la station en gazole, il faut organiser le transport du carburant vers ces lieux éloignés des centres urbains, et le plus souvent dénués d'infrastructures routières. La rétribution du mécanicien, comme l'achat et l'acheminement du carburant représentant des coûts de fonctionnement non négligeables, la question se posa d'emblée de savoir qui assumerait ces dépenses. Il ne s'agit plus là d'un simple problème technique ; c'est aussi une question d'ordre social.

Dans le dernier cas, celui d'un apport hydraulique sous pression, se posaient d'autres problèmes techniques. En principe, l'eau d'un puits artésien remonte d'elle-même en surface, en flux abondant, mais il 11) Thébaud, 1990, p. 29, note 14. 
faut ensuite régler la question de sa canalisation et de son utilisation optimale, pour abreuver les animaux et irriguer des jardins le cas échéant. L'entretien de cet équipement de surface devait d'abord être assuré, première difficulté, mais la plus grosse menace était celle de l'épuisement éventuel de l'eau. De nombreux forages se tarirent effectivement au bout d'un temps plus ou moins long, pour des raisons multiples : la pression de la nappe souterraine devenait insuffisante pour que l'artésianisme se maintienne, ou bien cette nappe s'épuisait en totalité, ou encore le sable bloquait la remontée de l'eau. Quoi qu'il en soit, ces installations n'ont pas eu, sur le long terme, le succès que l'on pouvait espérer et beaucoup durent être abandonnées.

Mais, au-delà des aspects techniques, c'est surtout du point de vue écologique et social que la mise en service de ces nouvelles installations provoqua de graves déséquilibres et des problèmes imprévus.

\section{Les déséquilibres écologiques}

Le milieu naturel sahélien est fragile. Sa préservation dépend d'un délicat équilibre qui doit s'opérer, en permanence, entre un pâturage, aléatoire dans le temps et dans l'espace, et le nombre d'animaux qui s'en nourrissent. Or l'hydraulique pastorale ne jouait que sur l'un des facteurs. Elle augmentait le nombre et le rendement des puits, autorisant donc la présence d'un nombre plus important d'animaux alors que le pâturage, quant à lui, restait le même puisque son abondance dépendait, comme auparavant, des aléas de la pluviométrie. Ces interventions étaient donc porteuses d'un risque de rupture de l'équilibre cheptel/pâturage, et les difficultés qui en résultèrent tiennent en deux mots : surcharge animale et désertification.

Les puits modernes présentaient cependant un avantage écologique important par rapport aux puits traditionnels. Comme ils ne nécessitent pas de coffrage, ils permettaient de faire une grosse économie de bois. En effet, non seulement les puits traditionnels sont généralement coffrés de bois, mais ils durent peu et il est nécessaire, tous les deux ou trois ans, d'en creuser un nouveau. A chaque fois, il faut abattre de nombreux arbres car les rondins du puits effondré ne sont pas récupérés. L'économie de cet abattage d'arbres était d'autant plus précieuse que le couvert arbustif est très long à se reconstituer. Mais 
cette économie ne compensait pas, néanmoins, la lourde menace sur le milieu que provoquait l'afflux de bétail dans ces zones écologiques fragiles. Les répercussions en furent variables selon les lieux et le type de puits.

Dans le cas des puits cimentés, les changements restaient minimes aux abords de chacun d'eux puisque le nombre d'animaux abreuvés demeurait subordonné à la lenteur de la remontée de l'eau. L'espacement des puits ( $24 \mathrm{~km}$ au moins) avait été calculé de façon à exploiter au mieux le pâturage disponible tout en limitant les risques de surcharge animale. En permettant une densité de cheptel bien plus forte sur des territoires jusqu'alors peu parcourus, cette politique favorisait indéniablement l'essor de l'élevage. Mais elle faisait reposer ses calculs de charge animale sur l'hypothèse d'une pluviométrie régulière et sous-estimait un aspect essentiel de l'économie pastorale sahélienne, à savoir le rôle stratégique des pâturages de réserve. En effet, même s'ils sont le plus souvent inoccupés, ces espaces jouent un rôle dans le pastoralisme sahélien. En période normale, ils peuvent servir de pâturages d'appoint et, en cas de sécheresse, ils constituent des espaces de repli vitaux pour le bétail.

Chaque année, les éleveurs sahéliens conduisent en effet une part ou la totalité de leurs troupeaux sur les espaces qui offrent le meilleur pâturage, parce qu'ils ont bénéficié d'une meilleure pluviométrie. Même les pasteurs les moins mobiles, qui campent la majeure partie de l'année aux abords d'un même puits, s'en éloignent au moins quelques mois par an, soit parce que le pâturage y manque à une période donnée, soit pour l'économiser car ils prévoient d'y revenir par la suite. D’autres éleveurs, plus itinérants, répètent chaque année plus ou moins les mêmes parcours, qui peuvent, comme dans l'est du Tchad, s'étendre sur plusieurs centaines de kilomètres ${ }^{12}$, et décident du mouvement de leurs troupeaux en fonction de l'état du pâturage, avec le même souci de préserver des espaces de repli quand les pâturages précédents sont épuisés. Le rôle de ces espaces peu occupés devient encore plus important en cas de crise climatique. 
De manière générale, la multiplication des puits, en permettant celle des troupeaux, fragilisait donc le pastoralisme car elle alourdissait, globalement, la charge animale sur des espaces aux ressources incertaines. Les risques s'en trouvaient aggravés en cas de sécheresse.

Les stations de pompage, en raison de leurs larges capacités d'abreuvage, concouraient encore plus à densifier le cheptel sur les espaces sahéliens, et donc à réduire les possibilités de repli en cas de crise. Mais elles provoquèrent surtout de graves difficultés à leurs abords immédiats. Car les éleveurs accoururent plus nombreux que prévu vers ces stations où l'eau se déversait dans les abreuvoirs sans aucun effort à faire. Finie, la dure corvée de l'abreuvage! Le rythme accéléré du remplissage des auges permettait, de plus, de faire boire un nombre bien plus grand d'animaux. Nombreux furent les pasteurs attirés par cette facilité. Il en résulta de très fortes concentrations de bétail autour de ces points d'eau, qui, rapidement, provoquèrent la désertification du pâturage, dans un cercle toujours plus large autour du puits. Le bétail dut ainsi parcourir des distances de plus en plus longues entre les lieux où il trouvait sa nourriture (les pâturages étant de plus en plus éloignés) et la station où il venait s'abreuver. Durant ces longs trajets, il perdait non seulement du temps, qu'il aurait mieux utilisé à paître, mais aussi de l'énergie, surtout lors des éprouvantes chaleurs d'avril et mai. Ces inconvénients ne tardèrent pas à l'emporter sur le bénéfice que procurait la facilité d'abreuvage. Les stations de pompage furent désertées par de nombreux éleveurs et leurs troupeaux.

Certaines stations furent définitivement fermées ou ouvertes seulement quelques mois par an, en rotation avec celles du voisinage, ou quelques heures par jour, afin de limiter ipso facto le nombre des animaux abreuvés sur place. La repousse du pâturage passait par cette réglementation stricte. En d'autres termes, la surcapacité technique de ces stations, pour l'abreuvage du bétail, rendait nécessaire la mise au point de règles sociales rigoureuses, afin d'éviter aussi bien la désertification du milieu que les conflits entre éleveurs. 


\section{Les problèmes sociaux}

Le bon fonctionnement de ces installations modernes soulevait de délicats problèmes d'organisation et de réglementation. Ces nouveaux points d'eau, créés en des lieux jusqu'alors non appropriés par les éleveurs, plaçaient en effet les usagers devant un vide juridique. Sur quelle base serait-il légitime de réglementer l'accès à l'eau, mais aussi aux pâturages environnants ? Pour envisager une réponse à cette question, il était utile d'abord de connaître la façon dont opéraient les systèmes pastoraux traditionnels.

Dans ces derniers, la remontée de l'eau restait à la charge de chaque éleveur. La question était donc simple : celui qui remonte l'eau en abreuve ses animaux, sous réserve toutefois qu'il ait le droit d'utiliser l'une des fourches du puits. En principe, tout éleveur qui se présente à un puits non occupé a le droit d'y lancer sa puisette et d'abreuver son bétail. Mais dans les usages anciens, en dépit des apparences, ce droit n'était pas pour autant laissé au hasard.

Le creusement et l'entretien des puits traditionnels étaient pris en charge, matériellement ou financièrement, par une famille ou un groupe de pasteurs. De ce fait, ceux-ci devenaient les propriétaires nominaux du puits, sans pour autant rester sur place ni utiliser le puits en permanence. Les aléas de la pluviométrie pouvaient les conduire sur d'autres lieux et d'autres parcours. Mais, lorsqu'ils revenaient à leur puits, ils avaient la priorité pour son usage. Les autres bergers de passage pouvaient l'utiliser mais devaient attendre, pour abreuver leurs troupeaux, que les usagers habituels leur aient laissé la place. Parfois, une rétribution modeste pouvait leur être demandée.

Cette souplesse du droit d'accès à l'eau est indispensable au Sahel. Elle garantit aux éleveurs une possibilité de mouvement qui permet d'optimiser l'exploitation des pâturages tout au long de l'année et conditionne la survie du bétail en cas de sécheresse. Cette liberté d'abreuver ses bêtes à différents puits relève donc de l'intérêt général de tous les pasteurs. 
Si l'ampleur et la régularité des mouvements pastoraux varient selon les lieux, les années et les habitudes de chaque groupe, beaucoup de familles gardent cependant pour point d'ancrage un puits auprès duquel elles résident le plus souvent, dont elles sont les propriétaires et usagers réguliers. Leurs droits de priorité, non seulement pour l'usage de l'eau mais aussi pour celui du pâturage environnant, sont parfois contestés, surtout si le pâturage se fait rare, mais ils font généralement l'objet de consensus.

Or ces habitudes préalables et ces droits d'usage n'existaient pas dans le cas des nouvelles installations de l'hydraulique pastorale et, de ce fait, deux questions cruciales se trouvaient sans réponse dans ce système : à qui appartiendraient ces puits ? Quelles priorités d'usage pourraient y être définies?

Pour les puits cimentés, le problème était simplifié par le nombre restreint des usagers. Deux cas de figure pouvaient se présenter : soit ce puits remplaçait un puits traditionnel préalable, soit il était creusé dans un nouvel espace non approprié.

Dans le premier cas, l'aménagement du puits se faisait avec l'accord des propriétaires des lieux. Le puits traditionnel préexistant était alors élargi ou recreusé, et cimenté. Sa plus grande largeur permettait d'y installer un ou deux fourches supplémentaires et donc d'abreuver un plus grand nombre d'animaux simultanément. Ces aménagements eurent pour effet de donner une plus grande permanence à l'habitat des éleveurs attachés à ces puits, sans changer de façon fondamentale les rapports sociaux puisque les familles auxquelles ils appartenaient en restaient les propriétaires. Le seul risque était d'inciter les éleveurs à restreindre leur mobilité, à rester sur place quand il pouvait être préférable de conduire le bétail ailleurs, en cas de raréfaction du pâturage.

Quant aux puits cimentés creusés dans des espaces non appropriés, ils n'étaient la propriété de personne. Ils pouvaient donc être utilisés par les premiers éleveurs venus, à charge pour eux d'en tirer l'eau. L'obligation d'attendre que d'autres, propriétaires attitrés du puits, se soient servis les premiers leur était épargnée, mais par contre ils 
devaient affronter la concurrence d'autres éleveurs, dénués comme eux d'un droit de priorité sur le puits. En cas de grande affluence, cette situation pouvait générer des tensions entre les usagers.

Sur les stations de pompage, le problème se posait en termes bien plus aigus encore. Non seulement la question du droit d'accès à l'eau n'était pas davantage tranchée, puisqu'aucun usage antérieur ne régissait des droits de priorité, mais, en outre, les candidats étaient fort nombreux à vouloir bénéficier de cette eau accessible sans effort. Les tensions entre éleveurs n'en étaient que plus fortes et le risque de conflit plus élevé. Il était alors impératif de réglementer les tours d'abreuvage. Mais il était plus simple d'énoncer le problème que de le résoudre, car il fallait pour cela définir des règles de priorité qui seraient admises par l'ensemble des éleveurs et assigner un responsable capable de les mettre en ouvre. Cet arbitre aurait pour rôle d'organiser les tours d'abreuvage, dans le respect de ces règles préalablement définies, afin d'éviter les conflits. Mais d'où cet arbitre tirerait-il sa légitimité, et de quelle façon envisageait-on de le rétribuer?

Par ailleurs, une autre question restait sans réponse : à qui reviendrait la charge de la maintenance de l'installation, notamment l'entretien et la fourniture de gazole, dans le cas d'une station de pompage ? Ces installations hydrauliques étaient des biens publics réalisés sur initiative administrative, et non à la demande des éleveurs. Il semblait donc normal à ces derniers que l'administration en assure l'entretien. Mais la grande pauvreté des pays sahéliens, et l'indigence de leurs administrations, ne pouvaient qu'entraver ou bloquer, sur le long terme, le suivi nécessaire au bon fonctionnement de ces installations. Il avait été plus facile de trouver de l'argent pour créer ces équipements, que pour en garantir ensuite un suivi régulier. Si énormes qu'ils pouvaient être, les problèmes techniques et écologiques entrâ̂nés par la création de ces équipements modernes semblaient donc moins inextricables que les difficultés juridiques et humaines qui se présentaient en même temps.

D'ailleurs, même lorsque le suivi technique et logistique était assuré, les nouvelles facilités d'abreuvage provoquaient une telle affluence et de tels engorgements autour de ces points d'eau (dans le cas des stations de pompage et des puits artésiens) que les problèmes ne fai- 
saient que s'additionner. Le plus grave, indéniablement, était la raréfaction du pâturage. La seule manière de s'y opposer était de limiter autoritairement - quand c'était possible - le temps d'ouverture de la station. Mais, en outre, la multitude des éleveurs et des troupeaux était source de confusion, de rixes, et les risques de vol de bétail s'en trouvaient accrus.

Cette situation dégénéra à un point tel que nombre d'éleveurs préférèrent renoncer au bénéfice d'un abreuvage facile et revenir, d'euxmêmes, au système préexistant. Au Niger par exemple, les Touaregs Illabakan demandèrent la fermeture d'une station de pompage installée dans leur région pour ne plus être gênés par les allées et venues des autres éleveurs. N'ayant pu obtenir gain de cause, ils choisirent de s'en écarter pour faire creuser plus loin à leurs frais, comme auparavant, leurs propres puits afin d'y jouir d'un droit incontestable d'usage prioritaire. Certes ils devaient tirer l'eau eux-mêmes, péniblement, par traction animale, mais ils étaient moins exposés au risque de voir leurs pâturages envahis par des étrangers : plus de travail sans doute, mais moins de concurrence, plus de tranquillité et moins de conflits ${ }^{13}$.

\section{Un bilan nuancé}

Le bilan de l'hydraulique pastorale, en dépit des sommes considérables qui y furent investies ${ }^{14}$, est donc loin d'être entièrement positif. Certes le creusement d'un grand nombre de nouveaux puits a permis l'exploitation de nouvelles et vastes surfaces de pâturage, et donc une augmentation très sensible du cheptel sahélien : en 30 ans, de 1940 à 1970, l'effectif bovin sahélien fut multiplié par $6^{15}$. L'abreuvage fut facilité. Enfin, la concentration humaine aux abords

13) Ce cas est rapporté par Bernus, 1992, p. 561.

14) Pierre Merlin indique qu'il obtenait "tout l'argent qu'il voulait" pour les nécessités du Service de l'hydraulique qu'il dirigeait (information orale du 12 décembre 2002).

15) Bernus, 1990, p. 273.

Afrique contemporaine - Printemps 2003 
des stations de pompage fut propice au développement de quelques petits marchés locaux, à la création d'écoles et de dispensaires, bien rares en milieu pastoral.

Mais, en contrepartie, ces installations modernes ont entraîné des problèmes, largement sous-estimés au départ, qui furent accentués par plusieurs années de sécheresse, à partir de 1969. Souvent ils ne purent être résolus. Tout d'abord, de nombreux puits artésiens ou stations de pompage sont devenus inopérants ${ }^{16}$, parfois pour de simples raisons techniques ou logistiques (épuisement de la nappe d'eau, ensablement, défaut de maintenance, manque de carburant). Les échecs ou les difficultés s'expliquent aussi par des raisons financières, sociales et psychologiques. Les instances administratives locales n'avaient souvent pas les moyens financiers, parfois pas la motivation suffisante pour prendre en charge les coûts de fonctionnement de ces installations. De leur côté, les éleveurs n'étaient pas davantage disposés à en assumer la charge, à moins d'une appropriation qui leur réserverait l'usage du puits.

En l'absence de propriétaires attitrés ou de l'autorité d'un arbitre reconnue de tous, seuls les rapports de force pouvaient régler la question de l'accès à l'eau et au pâturage environnant. Les conflits furent nombreux autour de ces points d'eau, en raison de la concurrence mutuelle des éleveurs. Le nombre des troupeaux rendait plus ardue la surveillance des animaux, et les voleurs de bétail ne manquèrent pas de profiter de la situation. Les risques de vol et de conflit représentaient des inconvénients graves pour les éleveurs, et pesaient lourd en contrepartie de l'avantage apporté par la facilité d'abreuvage, d'autant que la raréfaction du pâturage allongeait les trajets entre les pâturages et le point d'eau.

L'économie de l'abattage des arbres pour coffrer les puits était un avantage bien mince face à la surcharge animale sur les pâturages, et la dégradation de l'environnement (celle du couvert ligneux notamment) qu'elle provoquait. La raréfaction des arbres au Sahel est d'ailleurs, aujourd'hui, source d'une inquiétude croissante, car ces arbres ont des usages multiples : ils ne tiennent pas seulement lieu de

16) Au Nord-Sénégal par exemple, $46 \%$ des forages réalisés étaient hors service en 1982 selon Grenier, cité par Bernus, 1992, p. 560. 
pâturage pour les animaux, en complément du couvert herbacé, ils fournissent aussi à l'occasion du bois de construction, et surtout ils constituent la seule source d'énergie pour la cuisson des aliments. Aucune alternative opérationnelle, à ce jour, n'a encore été mise en place, auprès des populations sahéliennes, pour remplacer le bois de cuisson. Or, avec l'accroissement des villes, la demande urbaine de bois ou de charbon de bois est de plus en plus forte et, pour approvisionner les citadins, il faut le chercher de plus en plus loin.

Nous avons signalé également comment la densification animale qui résulte de ces installations hydrauliques ne provoque pas seulement la désertification autour des points d'eau à plus fort débit, mais réduit aussi les espaces de repli disponibles en cas de sécheresse. A cet égard, la situation des éleveurs sahéliens fut aggravée par l'extension toujours plus forte de la zone de cultures vers le nord, dès le début de la période coloniale. L'espace réservé aux pasteurs s'en trouvait de plus en plus rogné, au point qu'il fallut fixer administrativement une limite septentrionale à l'agriculture pour préserver les intérêts de

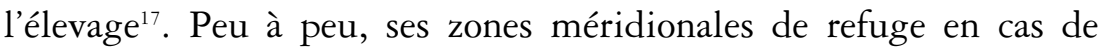
sécheresse se restreignaient, ce qui rendait ce dernier plus vulnérable aux aléas climatiques. C'est ainsi que l'on explique, notamment, le fait que les grandes sécheresses des années 1969-1974 puis des années 1980 (celles de 1983 et 1984 surtout) aient provoqué des catastrophes bien pires que les précédentes.

\section{Vers de nouveaux modes de vie}

Aujourd'hui, beaucoup d'éleveurs, qui sont descendus vers le sud lors d'une sécheresse, ont trouvé leur place auprès des populations locales et ne sont pas remontés vers le nord quand la pluviométrie y est redevenue plus clémente. Nombre d'entre eux sont devenus agro-pasteurs ou bien ont opéré une symbiose, plus ou moins réussie ${ }^{18}$, avec les agriculteurs sédentaires. A la demande des cultivateurs, les éleveurs conduisent leurs troupeaux sur les champs après la récolte. Les animaux enfument ainsi la terre, tout en se nourrissant des restes agri-

17) Au Niger par exemple, des textes législatifs furent promulgués en 1961 et 1962 pour fixer cette limite (Bernus, 1974, p. 122).

18) Arditi, 1999.

Afrique contemporaine - Printemps 2003 
coles laissés sur place. Les pasteurs se font payer pour cet enfumage, et trouvent en outre un marché pour le lait de leurs bêtes, qu'ils vendent aux agriculteurs ou aux citadins des villes voisines. Ainsi se créent de nouveaux modes de vie, se nouent de nouvelles transactions et de nouveaux contrats. Les agriculteurs sont de plus en plus nombreux à investir une part de leur revenu dans du bétail qu'ils confient aux éleveurs, et les pasteurs les plus démunis y trouvent un moyen d'existence. Beaucoup de ceux qui ont perdu tout leur bétail lors d'une sécheresse sont ainsi passés, au moins à titre provisoire, du statut de propriétaire à celui de berger pour le compte d'autrui en attendant d'être en mesure de reconstituer leurs propres troupeaux. Prolétarisation d'un côté, enrichissement par la vente du lait de l'autre, ou sédentarisation plus ou moins durable des éleveurs qui deviennent cultivateurs en attendant un retour hypothétique à la vie pastorale, toujours est-il que la coupure radicale qui opposait agriculteurs sédentaires et pasteurs nomades prend, peu à peu, une allure moins tranchée ${ }^{19}$.

\section{Attribuer les points d'eau}

Cependant, dans les zones septentrionales du Sahel, qui par leur faible pluviométrie restent exclusivement réservées à l'élevage, les spécialistes du développement sont davantage conscients aujourd'hui de la nécessité d'attribuer les points d'eau modernes à des groupes ou des associations pastorales susceptibles d'en assurer la gestion ${ }^{20}$. Les leçons du passé ont bien montré que laisser ces installations dans le domaine public ne conduit qu'à une détérioration toujours plus forte des espaces pastoraux. Ce changement de statut juridique des points d'eau est, selon les termes de Brigitte Thébaud, spécialiste du développement pastoral sahélien, "l'une des conditions essentielles à la réhabilitation des systèmes pastoraux sahéliens, là où il n'est pas encore trop tard pour la faire ${ }^{211}$. Mais ce changement suppose d'abord que d'importantes réformes soient mises en œuvre par les gouvernements sahéliens, en matière de droit foncier et de code rural ${ }^{22}$. C'est dire combien de difficultés restent à surmonter. 
La triste histoire de ces 40 années d'expérience en matière d'hydraulique pastorale, des années 1960 à nos jours, aura eu au moins le mérite de mettre en évidence la remarquable adaptation non seulement des pratiques, mais aussi du droit traditionnel des sociétés pastorales sahéliennes aux particularités de leur milieu. Les spécialistes du développement pastoral prônent aujourd'hui un retour vers une forme approchée de ce droit. Cette histoire illustre aussi - faut-il le souligner? - combien les avancées technologiques sont vaines, si les facteurs humains ne sont pas, d'abord, pris en compte.

\section{Références bibliographiques}

Arditi, C. , "Paysans sara et éleveurs arabes dans le sud du Tchad : du conflit à la cohabitation ?", dans C. Baroin et J. Boutrais (dir.), L'Homme et l'animal dans le bassin du lac Tchad, Paris, Institut de recherche pour le développement (IRD), 1999, p. 555-573.

Bernus, E., "Possibilités et limites de la politique d'hydraulique pastorale dans le Sahel nigérien", Cabiers de sciences humaines de l'ORSTOM, vol. 11, n 2, 1974, p. $119-126$

Bernus, E., "L'eau du désert. Usages, techniques et maîtrise de l'espace aux confins du Sahara", Etudes rurales, n $115-116,1989$, p. 93-104.

Bernus, E., "Hydraulique pastorale et gestion des parcours", dans E. Le Floc'h, M. Grouzis, A. Cornet et J.-C. Bille (dir.), L'A ridité : une contrainte au développement. Caractérisation, réponses biologiques, stratégies des sociétés, Paris, ORSTOM, 1992, p. 555-563.

Bernus, E., "Les pasteurs nomades africains, du mythe éternel aux réalités présentes", dans E. Bernus et J. Pouillon (dir.), "Sociétés pastorales et développement", Cabiers de sciences humaines de l'ORSTOM, vol. 26, n 1-2, 1990, p. 267-280.

Bernus, E. et J. Pouillon (dir.), "Sociétés pastorales et développement", Cabiers de sciences humaines de l'ORSTOM, vol. 26, n 1-2, 1990.

Bonfiglioli, A.M., "Pastoralisme, agro-pastoralisme et retour : itinéraires sahéliens", dans E. Bernus et J. Pouillon (dir.), "Sociétés pastorales et développement", Cabiers de sciences humaines de l'ORSTOM, vol. 26, n 1-2, 1990, p. 255-266.

Botte, R. (dir.), Journal des africanistes, numéro spécial "L'ombre portée de l'esclavage. Avatars contemporains de l'oppression sociale", vol. 70, n 1-2, 2000.

Afrique contemporaine - Printemps 2003 
Clanet, J.-C., "Structures spatiales et cultures pastorales (ou les limites des Etatsnations et des organisations tribales en Afrique centrale)", dans C. Baroin et J. Boutrais (dir.), L'Homme et l'animal dans le bassin du lac Tchad, Paris, IRD, 1999, p. 649-666.

Landais, E., "Sur les doctrines des vétérinaires coloniaux français en Afrique noire", dans E. Bernus et J. Pouillon (dir.), "Sociétés pastorales et développement", Cabiers de sciences bumaines de l'ORSTOM, vol. 26, n 1-2, 1990, p. 33-71.

Meillassoux, C. (dir.), L'Esclavage en Afrique précoloniale, Paris, Maspéro, 1975.

Merlin, P., L'Hydraulique pastorale en A.O.F. Rapport présenté à la Conférence des Chefs de Service de l'élevage de l'A.O.F., Bamako (Soudan français), 22-27 janvier 1951.

Merlin, P., Espoir pour l'Afrique noire, Paris, Présence africaine, 1996.

Receveur, P., "Politique de mise en valeur de la zone sahélienne", Rapports annuels, République du Sénégal, ministère de l'Economie rurale, Direction de l'élevage et des industries animales, 1959-1960, chap. I à IX, p. 129-150.

Receveur, P., Définition d'un programme d'aménagements hydropastoraux dans la zone sylvo-pastorale, République du Sénégal, ministère de l'Economie rurale, Service de l'élevage, 1965.

Receveur, P., Aménagements pastoraux en zone sabélienne, Maisons-Alfort, Institut d'élevage et de médecine vétérinaire des pays tropicaux (IEMVT), 1975.

Thébaud, B., "L'évolution récente des politiques d'hydraulique pastorale et la gestion de l'espace au Sahel", dans E. Bernus et J. Pouillon (dir.), "Sociétés pastorales et développement", Cabiers de sciences humaines de l'ORSTOM, vol. 26, n 1-2, 1990, p. 97-117.

Thébaud, B., Foncier pastoral et gestion de l'espace au Sabel. Peuls du Niger oriental et du Yagha burkinabè, Paris, Karthala. 